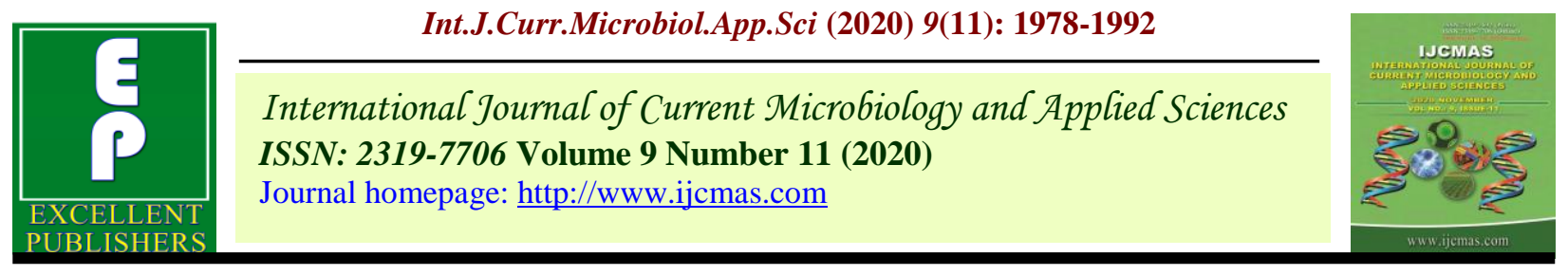

Original Research Article

https://doi.org/10.20546/ijcmas.2020.911.235

\title{
DUS Characterization of Field Pea Genotypes (Pisum sativum L.) Using Morphological Markers
}

\author{
Theint Theint Tun, Ravika*, Rajesh Yadav and Suman \\ Department of Genetics and Plant Breeding, CCS Haryana Agricultural University, \\ Hisar-125004, India \\ *Corresponding author
}

\begin{abstract}
A B S T R A C T
Keywords

Field pea, DUS

characterization

Article Info

Accepted:

15 October 2020

Available Online:

10 November 2020

In field pea germplasm a wide range of genetic variability is present for various morphological traits; In this regard, present genotypes were dimly characterized for these morphological descriptors to establish DUS. DUS test is important so that new varieties can achieve legal access to their market or their protection under PPV\&FR Act, 2001. The present paper focuses on DUS characterization for 126 genotypes of field pea with diverse origin including four checks viz., HFP 8909, HFP 715, HFP 529 and HFP 9426 grown in Augmented Design. The study was carried out during Rabi season 2018-19. 20 morphological characters were recorded as per National Test Guidelines for DUS Testing on field pea.
\end{abstract}

\section{Introduction}

In India, Pulses holds important position among food grains as majority of population is vegetarian and depends on pulses for protein supplement. Besides being rich source of protein, they are also vital for sustainable agriculture and also fix atmospheric nitrogen through symbiosis making them exceptionally valuable both economically and nutritionally. Growing legumes improves soil quality through their beneficial effects on its biological, chemical and physical properties. Fieldpea (Pisum sativum L.) is a rabi season self-pollinated diploid crop $(2 \mathrm{n}=14)$ belongs to family Fabaceae. It is the third most widely grown food legume worldwide (Tyagi et al., 2012). In India, field pea is grown over an area of $0.82 \mathrm{~m}$ ha, with production of $0.99 \mathrm{~m}$ tonnes and productivity $1205 \mathrm{~kg} / \mathrm{ha}$ (Anonymous, 2019). It is truly a multifunctional crop that may be used as green forage, forage dry matter, forage meal, silage, haylage, immature grain, mature grain, straw and green manure (Mihailovic and Mikic, 2010). Field pea is naturally dried one that are consumed as dhal, soups, stews and various other cuisines. Field pea is primarily valued for the nutritional quality of its seeds which have high nutritional grade for resource poor 
families (Nawab et al., 2008). Dry seeds of fieldpea contain $22.9 \%$ protein, $60.7 \%$ carbohydrates, $1.45 \%$ fat, $1.4 \%$ crude fiber and $2.7 \%$ ash with calorific value $343 / 100 \mathrm{~g}$ (Duke, 1981).

As demand of pulses is increasing but growing area is limited, there is urgent need to enhance productivity per unit area and time. Parents selected with desirable traits and their use in hybridization programme is of paramount importance. It is well established that yield is a complex trait and inclusion of all the component traits in selection scheme for such intricate characters is impractical.

Characterization makes an easy and quick difference among various accessions, which can be easily accessed later for particular traits of interest. Usually the descriptions provided by the plant breeders are generally inadequate to characterize a genotype. Therefore, it is imperative to characterize the genotypes and to identify the genotypic purity. DUS testing is a way of determining whether a genotype differs from others within the same species (the Distinctness part), whether the characteristics used to establish Distinctness are expressed uniformly (the Uniformity part) and that these characteristics do not change over subsequent generations (the Stability part). The DUS testing principles were used for the characterization of 126 fieldpea genotypes for twenty traits as per the Guidelines for the conduct of test for Distinctiveness, Uniformity and Stability on fieldpea. Therefore, the present study was formulated to have a better knowledge about the composition and diversity of the accessions by establishing distinctness in field pea lines as per National Guidelines for DUS testing.

\section{Materials and Methods}

The field experiment was carried out during Rabi season 2018-2019 at Pulses Research
Area, Department of Genetics and Plant Breeding, CCS Haryana Agricultural University, Hisar, which is situated in semiarid sub-tropical region at 29o10' $\mathrm{N}$ latitude and 75046' E longitude with elevation of $215.52 \mathrm{~m}$ above mean sea level. The experimental material included 126 genotypes of diverse origin and four checks viz., HFP 8909, HFP 715, HFP 529 and HFP 9426. Genotypes were grown in Augmented Design with five blocks and each genotype was grown in a single row of $4 \mathrm{~m}$ length with 45 and $10 \mathrm{~cm}$ spacing. First four blocks comprised of 25 genotypes each and the fifth block was sown with 26 genotypes with repetition of checks in all the five blocks. The experimental soil was sandy loam and all the recommended package of practices was followed to raise a good crop. Guidelines for the conduct of test for DUS on Field pea as per PPV\&FRA, Government of India (Anonyms 2007) were followed to evaluate field zpea genotypes for 20 characters namely, Stem anthocyanin coloration, foliage colour, leaf leaflets, stipule rabbit-eared stipule, stipule type, foliage waxi bloom, leaf axil color, time of flowering, flower standard petal color, number of pods per axil, pod curvature, pod shape of distal part, pod intensity of green color, plant height, seed shape, seed surface, seed cotyledon color, 100 -seed weight, seed testa mottling and seed parchment.

The characters were observed as visual assessment by a single observation of a group of plants or parts of plants at the specific stage of the crop. Stem anthocyanin coloration, foliage colour, leaf leaflets, stipule rabbiteared stipule, stipule type, foliage waxi bloom and leaf axil color were recorded at initiation of flowering. Flower opening when 50 per cent of the plants with at least one open flower; flower standard petal color at 50 per cent flowering. Number of pods per axil, pod curvature, pod shape of distal part, pod intensity of green color and seed parchment 
were recorded at fully developed green pod stage. At seed maturity stage, seed shape, seed surface, seed cotyledon color, 100-seed weight and seed testa mottling were observed.

Characters foliage colour, foliage waxy bloom, leaflets, rabbit eared stipules, stipule type, flower opening, flower standard petal colour, pod curvature, pod intensity of green colour, seed shape, seed surface, seed cotyledon colour, seed testa mottling and seed parchment were recorded by visual assessment by a single observation of a group of plants or parts of plants whereas, visual assessment by observation of individual plants or parts of plants was used to observe stem anthocyanin coloration, leaf axil colour, pod number per axil and pod shape of distal.

\section{Results and Discussion}

Dendrogram (Fig. 1) exhibits association among the different genotypes and it was prepared with rescaled distances using SPSS software.

In dendrogram the genotypes lying near to each other are more similar to one another than those lying apart. The resemblance coefficient between the two genotypes is the value at which their branches join. The dendrogram exhibited the relative magnitude of resemblance among the different clusters. State and frequency distribution of fieldpea genotypes for different descriptors is depicted in Table 1. The fieldpea genotypes exhibited considerable variation for the majority of characters under study except stipule type, Seed testa mottling and seed perchmant. Singh et al., (2014) while characterizing vegetable pea varieties on the basis of 19 descriptors also reported considerable variation for all the important attributes except anthocyanin colouration, leaf axil colour and standard petal colour.
In our investigation all the groups, as described in DUS Guidelines for Fieldpea, were observed for stem anthocyanin colouration, foliage colour, leaf: leaflets, rabbit-eared stipules, foliage waxy bloom, pod curvature, pod shape of distal part, seed shape, seed surface and 100-seed weight in the 126 genotypes studied. For time of flowering two out of four, for flower colour two out of five and for number of pods per axil, pod intensity of green colour, plant height and seed cotyledon colour only two out of three groups were observed. No variability for stipule type, seed testa mottling and seed parchment was observed in the present material indicating presence of sufficient amount of genetic variability among these genotypes for almost all the traits studied. As per the DUS guidelines, the characters viz., plant height, rabbit-eared stipules, time of flowering, pod shape of distal part, seed cotyledon colour and seed shape should be used for grouping of genotypes and in our study considerable amount of variability for all these traits was observed. Dixit et al., (2010), Singh et al., (2014), Ouafi et al., (2016) and Yadav et al., (2019) characterized and grouped a large number of fieldpea genotypes for various morphological parameters viz. stem anthocyanin coloration, foliage colour, leaf/ leaflets type, rabbit eared stipule, stipule type, leaf juncture colour, flowering time, plant type, flower colour, number of pods/axil, pod shape, pod beak, seed shape, cotyledon colour, seed size and seed testa mottling, etc.

Observations on stem anthocyanin coloration revealed its presence only in 11 genotypes (DDR-4, EC 398601, EC 412882, IP2K 83-1, IPF 11-15, NGSN-10, NGSN-11, NGSN-13, NGSN-6, Pant P-2009-3, RFP 2009-2), however, in other 115 genotypes it was absent. For character foliage colour genotypes were grouped as light green, green and dark green. Out of the 126 genotypes studied, 16 genotypes were having light green foliage 
colour (20/1062, DMR-7, HFP 0110, HFP 0145, HFP 0149, HFP 1104, HFP 9106, HFP 920, IPF 11-13, IPF 5-19, KPF 115, NGSN14, Pant P-215, Pant P-217, Pant P-42, RFP 2010-3), 83 genotypes were having green and 27 genotypes were having dark green foliage colour.

Leaf leaflets, absent in 14 genotypes (Aman, HFP 1036, HFP 1125, HFP 1302, HFP 1307, HUPT 1701, IPFD 12-2, KFP 12-4, KPF 1435, KPMR 928, Pant P-222, RFPG 114, SPC101, VL-202) and present in 112 genotypes. Out of 126 genotypes, in 106 genotypes rabbit-eared stipule was absent and in rest of the 20 (Ambika, DMR-7, EC 412882, HFP 1129, IP2K 83-1, IPFD 201411, KFP 12-4, KPMR 928, NDP 14-11, NGSN-10, NGSN-5, NGSN-6, NGSN-8, Pant P-195, Pant P-200, Pant P-222, Pant P-223, Pant P-247, PH-1, VL-202) genotypes it was present.

For stipule type, genotypes were grouped in two groups viz., normal and vestigial in our investigation only one group was observed i.e. all the genotypes were of normal type.

In 92 genotypes foliage waxy bloom was absent and in rest of the 34 (Aman, EC 398601, EC 412882, HFP 1036, HFP 1107, HFP 1125, HFP 1129, HFP 1132, HFP 1137, HFP 1302, HFP 1307, HFP 1314, HFP 1315, HFP 1405, HFP 2008, HFP 530B, HFP 9907B, HUDP 1302, HUDP 1715, HUPT 1701, IPFD 12-2, IPFD 17-6, KFP 12-4, KPMR 928, NGSN-10, NGSN-11, NGSN-6, Pant P-215, Pant P-250, Pant P-365, Pant P367, RFPG 114, SPC101, VL-55) genotypes it was present.

115 genotypes were observed green leaf axil colour and remaining 11 genotypes were found purple. Four groups can be determined for time of flowering viz., extra early $(<40)$, early (40-50), medium (51-70) and late (>70) on the basis of DUS guidelines for fieldpea, however, in our results only two groups i.e. medium and late were recorded. Among the 126 genotypes, 14 genotypes were found medium (Ambika, DDR-4, HFP 1104, HFP 9907A, HUDP 1301, HUDP 1302, IPF 5-19, NGSN-10, NGSN-11, NGSN-13, Pant P-399, Pant P-402, PH-1, RFP 72) and 112 genotypes were found late. Singh et al., (2014) also observed all the four classes and stipulated that short duration varieties are often less sensitive to photoperiod than the late maturing ones. Out of the five classes of petal colour (standard), most of the genotypes were white petaled except 11 (DDR-4, EC 398601, EC 412882, IP2K 83-1, IPF 11-15, NGSN-10, NGSN-11, NGSN-13, NGSN-6, Pant P-2009-3, RFP 2009-2) which had purple. None of the genotypes was observed with blue, pink and red coloured petals. While studying 51 fieldpea genotypes, Bhuvaneswari et al., (2017) also observed majority of the genotypes with white flowers. Furthermore, Yadav et al., (2019) grouped fieldpea genotypes on the basis of anthocyanin coloration of axil, stipule and color of standard. Only six genotypes (DDR4, HFP 0130, IP2K 83-1, KPMR 745, NDP 11-101, NGSN-3) bore single pod per axil while the rest 120 genotypes had double.

Pod curvature was absent only in one genotype (EC 412882), 84 genotypes had weak, 40 genotypes had medium and only one genotype was having strong curvature (NGSN-10).

Out of the 126 genotypes studied, 63 genotypes were having pointed and rest of the 63 genotypes was having blunt shape of distal part. While pod shape of distal part was found pointed in 63 genotypes and blunt in 63 . Singh et al., (2014) also found single pod/axil and double pod/axil in their study. In respect of pod curvature, they found only three classes, however, both pointed and blunt type of pod shape of distal end were observed by them. 
Fig.1 Dendrogram representing relatedness among the fieldpea genotypes

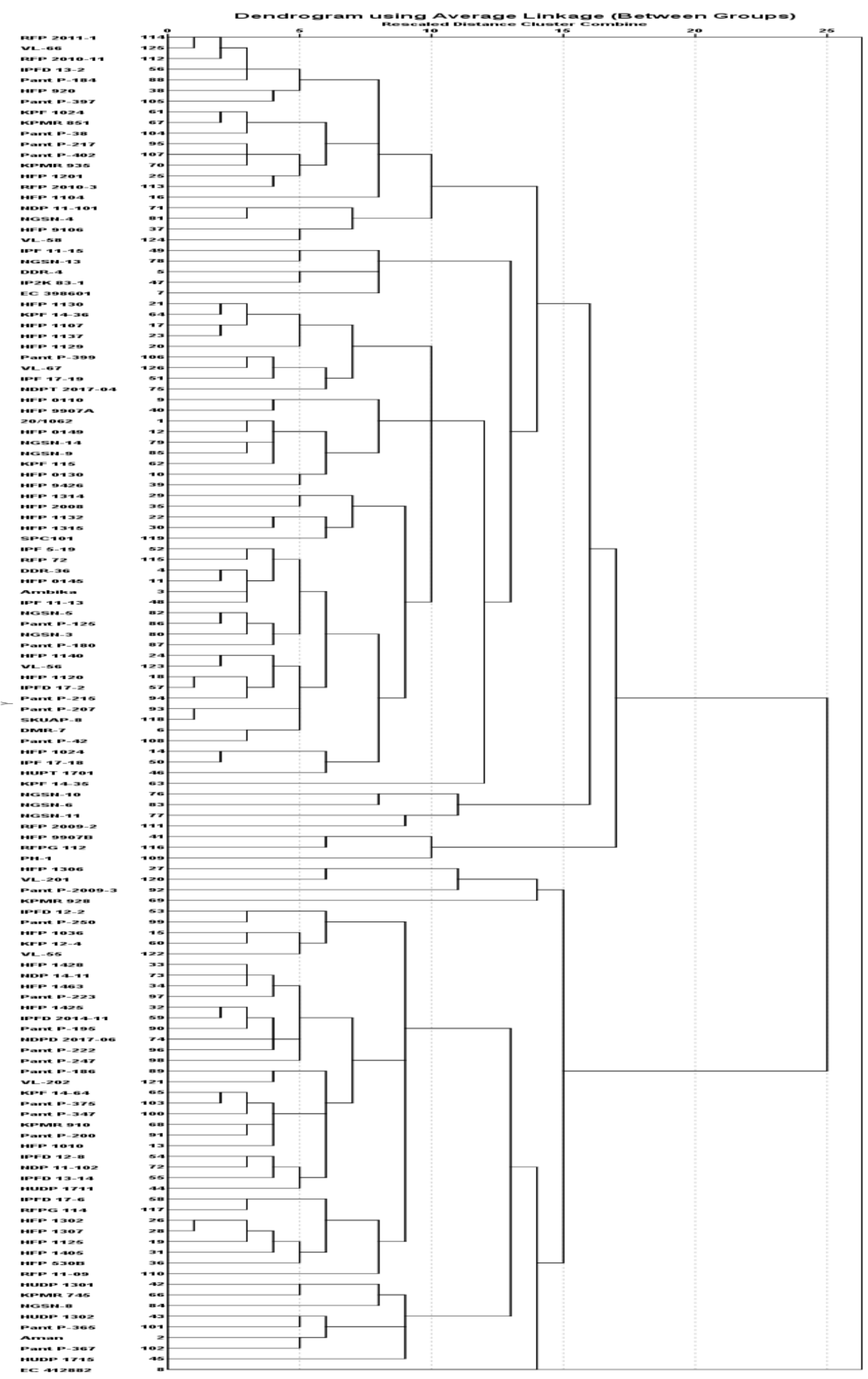


Table.1 State and frequency distribution of fieldpea genotypes for different descriptors

\begin{tabular}{|c|c|c|c|c|}
\hline $\begin{array}{l}\text { S. } \\
\text { No. }\end{array}$ & Traits & State & Genotypes & $\begin{array}{c}\text { No. of } \\
\text { genotypes }\end{array}$ \\
\hline \multirow[t]{2}{*}{1.} & \multirow[t]{2}{*}{$\begin{array}{l}\text { Stem: Anthocyanin } \\
\text { colouration }\end{array}$} & Absent: 1 & $\begin{array}{l}\text { 20/1062, Aman, Ambika, DDR-36, DMR-7, HFP 0110, HFP 0130, HFP 0145, HFP 0149, HFP 1010, HFP 1024, } \\
\text { HFP 1036, HFP 1104, HFP 1107, HFP 1120, HFP 1125, HFP 1129, HFP 1130, HFP 1132, HFP 1137, HFP 1140, } \\
\text { HFP 1201, HFP 1302, HFP 1306, HFP 1307, HFP 1314, HFP 1315, HFP 1405, HFP 1425, HFP 1428, HFP 1463, } \\
\text { HFP 2008, HFP 530B, HFP 9106, HFP 920, HFP 9426, HFP 9907A, HFP 9907B , HUDP 1301, HUDP 1302, } \\
\text { HUDP 1711, HUDP 1715, HUPT 1701, IPF 11-13, IPF 17-18, IPF 17-19, IPF 5-19, IPFD 12-2, IPFD 12-8, IPFD } \\
\text { 13-14, IPFD 13-2, IPFD 17-2, IPFD 17-6, IPFD 2014-11, KFP 12-4, KPF 1024, KPF 115, KPF 14-35, KPF 14-36, } \\
\text { KPF 14-64, KPMR 745, KPMR 851, KPMR 910, KPMR 928, KPMR 935, NDP 11-101, NDP 11-102, NDP 14- } \\
\text { 11, NDPD 2017-06, NDPT 2017-04, NGSN-14, NGSN-3, NGSN-4, NGSN-5, NGSN-8, NGSN-9, Pant P-125, } \\
\text { Pant P-180, Pant P-184, Pant P-186, Pant P-195, Pant P-200, Pant P-207, Pant P-215, Pant P-217, Pant P-222, Pant } \\
\text { P-223, Pant P-247, Pant P-250, Pant P-347, Pant P-365, Pant P-367, Pant P-375, Pant P-38, Pant P-397, Pant P- } \\
\text { 399, Pant P-402, Pant P-42, PH-1, RFP 11-09, RFP 2010-11, RFP 2010-3, RFP 2011-1, RFP 72, RFPG 112, } \\
\text { RFPG 114, SKUAP-8, SPC101, VL-201, VL-202, VL-55, VL-56, VL-58, VL-66, VL-67 }\end{array}$ & 115 \\
\hline & & Present:9 & $\begin{array}{l}\text { DDR-4, EC 398601, EC 412882, IP2K 83-1, IPF 11-15, NGSN-10, NGSN-11, NGSN-13, NGSN-6, Pant P-2009- } \\
\text { 3, RFP 2009-2 }\end{array}$ & 11 \\
\hline \multirow[t]{3}{*}{2.} & \multirow[t]{3}{*}{ Foliage: Colour } & $\begin{array}{l}\text { Light } \\
\text { green:3 }\end{array}$ & $\begin{array}{l}\text { 20/1062, DMR-7, HFP 0110, HFP 0145, HFP 0149, HFP 1104, HFP 9106, HFP 920, IPF 11-13, IPF 5-19, KPF } \\
\text { 115, NGSN-14, Pant P-215, Pant P-217, Pant P-42, RFP 2010-3 }\end{array}$ & 16 \\
\hline & & Green:5 & $\begin{array}{l}\text { Aman, Ambika, DDR-36, DDR-4, EC 398601, EC 412882, HFP 1024, HFP 1107, HFP 1120, HFP 1129, HFP } \\
\text { 1130, HFP 1132, HFP 1137, HFP 1140, HFP 1201, HFP 1306, HFP 1314, HFP 1315, HFP 1405, HFP 1425, HFP } \\
\text { 1428, HFP 1463, HFP 2008, HFP 9426, HFP 9907A, HFP 9907B , HUDP 1301, HUDP 1302, HUDP 1711, } \\
\text { HUDP 1715, HUPT 1701, IP2K 83-1, IPF 11-15, IPF 17-18, IPF 17-19, IPFD 12-8, IPFD 13-14, IPFD 13-2, IPFD } \\
\text { 17-2, IPFD 2014-11, KPF 14-36, KPF 14-64, KPMR 745, KPMR 910, KPMR 935, NDP 11-101, NDP 11-102, } \\
\text { NDP 14-11, NDPD 2017-06, NDPT 2017-04, NGSN-10, NGSN-11, NGSN-13, NGSN-8, Pant P-125, Pant P-180, } \\
\text { Pant P-184, Pant P-186, Pant P-195, Pant P-200, Pant P-2009-3, Pant P-207, Pant P-347, Pant P-365, Pant P-375, } \\
\text { Pant P-397, Pant P-399, Pant P-402, PH-1, RFP 11-09, RFP 2009-2, RFP 2010-11, RFP 2011-1, RFP 72, RFPG } \\
\text { 112, RFPG 114, SKUAP-8, SPC101, VL-202, VL-56, VL-58, VL-66, VL-67 }\end{array}$ & 83 \\
\hline & & Dark green:7 & $\begin{array}{l}\text { HFP 0130, HFP 1010, HFP 1036, HFP 1125, HFP 1302, HFP 1307, HFP 530B, IPFD 12-2, IPFD 17-6, KFP 12-4, } \\
\text { KPF 1024, KPF 14-35, KPMR 851, KPMR 928, NGSN-3, NGSN-4, NGSN-5, NGSN-6, NGSN-9, Pant P-222, } \\
\text { Pant P-223, Pant P-247, Pant P-250, Pant P-367, Pant P-38, VL-201, VL-55 }\end{array}$ & 27 \\
\hline \multirow[t]{2}{*}{3.} & \multirow[t]{2}{*}{ Leaf: Leaflets } & Absent: 1 & $\begin{array}{l}\text { Aman, HFP 1036, HFP 1125, HFP 1302, HFP 1307, HUPT 1701, IPFD 12-2, KFP 12-4, KPF 14-35, KPMR 928, } \\
\text { Pant P-222, RFPG 114, SPC101, VL-202 }\end{array}$ & 14 \\
\hline & & Present:9 & $\begin{array}{l}\text { 20/1062, Ambika, DDR-36, DDR-4, DMR-7, EC } 398601 \text {, EC } 412882 \text {, HFP 0110, HFP 0130, HFP 0145, HFP } \\
\text { 0149, HFP 1010, HFP 1024, HFP 1104, HFP 1107, HFP 1120, HFP 1129, HFP 1130, HFP 1132, HFP 1137, HFP } \\
\text { 1140, HFP 1201, HFP 1306, HFP 1314, HFP 1315, HFP 1405, HFP 1425, HFP 1428, HFP 1463, HFP 2008, HFP } \\
\text { 530B, HFP 9106, HFP 920, HFP 9426, HFP 9907A, HFP 9907B , HUDP 1301, HUDP 1302, HUDP 1711, HUDP } \\
\text { 1715, IP2K 83-1, IPF 11-13, IPF 11-15, IPF 17-18, IPF 17-19, IPF 5-19, IPFD 12-8, IPFD 13-14, IPFD 13-2, }\end{array}$ & 112 \\
\hline
\end{tabular}




\begin{tabular}{|c|c|c|c|c|}
\hline & & & $\begin{array}{l}\text { IPFD 17-2, IPFD 17-6, IPFD 2014-11, KPF 1024, KPF 115, KPF 14-36, KPF 14-64, KPMR 745, KPMR 851, } \\
\text { KPMR 910, KPMR 935, NDP 11-101, NDP 11-102, NDP 14-11, NDPD 2017-06, NDPT2017-04, NGSN-10, } \\
\text { NGSN-11, NGSN-13, NGSN-14, NGSN-3, NGSN-4, NGSN-5, NGSN-6, NGSN-8, NGSN-9, Pant P-125, Pant P- } \\
\text { 180, Pant P-184, Pant P-186, Pant P-195, Pant P-200, Pant P-2009-3, Pant P-207, Pant P-215, Pant P-217, Pant P- } \\
\text { 223, Pant P-247, Pant P-250, Pant P-347, Pant P-365, Pant P-367, Pant P-375, Pant P-38, Pant P-397, Pant P-399, } \\
\text { Pant P-402, Pant P-42, PH-1, RFP 11-09, RFP 2009-2, RFP 2010-11, RFP 2010-3, RFP 2011-1, RFP 72, RFPG } \\
\text { 112, SKUAP-8, VL-201, VL-55, VL-56, VL-58, VL-66, VL-67 }\end{array}$ & \\
\hline \multirow[t]{2}{*}{4.} & \multirow[t]{2}{*}{$\begin{array}{l}\text { Stipule: Rabbit- } \\
\text { eared stipules }\end{array}$} & Absent:1 & $\begin{array}{l}\text { 20/1062, Aman, DDR-36, DDR-4, EC 398601, HFP 0110, HFP 0130, HFP 0145, HFP 0149, HFP 1010, HFP } \\
\text { 1024, HFP 1036, HFP 1104, HFP 1107, HFP 1120, HFP 1125, HFP 1130, HFP 1132, HFP 1137, HFP 1140, HFP } \\
\text { 1201, HFP 1302, HFP 1306, HFP 1307, HFP 1314, HFP 1315, HFP 1405, HFP 1425, HFP 1428, HFP 1463, HFP } \\
\text { 2008, HFP 530B, HFP 9106, HFP 920, HFP 9426, HFP 9907A, HFP 9907B , HUDP 1301, HUDP 1302, HUDP } \\
\text { 1711, HUDP 1715, HUPT 1701, IPF 11-13, IPF 11-15, IPF 17-18, IPF 17-19, IPF 5-19, IPFD 12-2, IPFD 12- } \\
\text { 8,IPFD 13-14,IPFD 13-2,IPFD 17-2,IPFD 17-6, KPF 1024, KPF 115, KPF 14-35, KPF 14-36, KPF 14-64, KPMR } \\
\text { 745, KPMR 851, KPMR 910, KPMR 935, NDP 11-101, NDP 11-102, NDPD 2017-06, NDPT 2017-04, NGSN- } \\
\text { 11, NGSN-13, NGSN-14, NGSN-3, NGSN-4, NGSN-9, Pant P-125, Pant P-180, Pant P-184, Pant P-186, Pant P- } \\
\text { 2009-3, Pant P-207, Pant P-215, Pant P-217, Pant P-250, Pant P-347, Pant P-365, Pant P-367, Pant P-375, Pant P- } \\
\text { 38, Pant P-397, Pant P-399, Pant P-402, Pant P-42, RFP 11-09, RFP 2009-2, RFP 2010-11, RFP 2010-3, RFP } \\
\text { 2011-1, RFP 72, RFPG 112, RFPG 114, SKUAP-8, SPC101, VL-201, VL-55, VL-56, VL-58, VL-66, VL-67 }\end{array}$ & 106 \\
\hline & & Present:9 & $\begin{array}{l}\text { Ambika, DMR-7, EC 412882, HFP 1129, IP2K 83-1, IPFD 2014-11, KFP 12-4, KPMR 928, NDP 14-11, NGSN- } \\
\text { 10, NGSN-5, NGSN-6, NGSN-8, Pant P-195, Pant P-200, Pant P-222, Pant P-223, Pant P-247, PH-1, VL-202 }\end{array}$ & 20 \\
\hline \multirow[t]{2}{*}{5.} & \multirow[t]{2}{*}{ Stipule: Type } & Normal:1 & $\begin{array}{l}\text { 20/1062, Aman, Ambika, DDR-36, DDR-4, DMR-7, EC 398601, EC 412882, HFP 0110, HFP 0130, HFP 0145, } \\
\text { HFP 0149, HFP 1010, HFP 1024, HFP 1036, HFP 1104, HFP 1107, HFP 1120, HFP 1125, HFP 1129, HFP 1130, } \\
\text { HFP 1132, HFP 1137, HFP 1140, HFP 1201, HFP 1302, HFP 1306, HFP 1307, HFP 1314, HFP 1315, HFP 1405, } \\
\text { HFP 1425, HFP 1428, HFP 1463, HFP 2008, HFP 530B, HFP 9106, HFP 920, HFP 9426, HFP 9907A, HFP } \\
\text { 9907B, HUDP 1301, HUDP 1302, HUDP 1711, HUDP 1715, HUPT 1701, IP2K 83-1, IPF 11-13, IPF 11-15, IPF } \\
\text { 17-18, IPF 17-19, IPF 5-19, IPFD 12-2, IPFD 12-8, IPFD 13-14, IPFD 13-2, IPFD 17-2, IPFD 17-6, IPFD 2014- } \\
\text { 11, KFP 12-4, KPF 1024, KPF 115, KPF 14-35, KPF 14-36, KPF 14-64, KPMR 745, KPMR 851, KPMR 910, } \\
\text { KPMR 928, KPMR 93,, NDP 11-101, NDP 11-102, NDP 14-11, NDPD 2017-06, NDPT 2017-04, NGSN-10, } \\
\text { NGSN-11, NGSN-13, NGSN-14, NGSN-3, NGSN-4, NGSN-5, NGSN-6, NGSN-8, NGSN-9, Pant P-125, Pant P- } \\
\text { 180, Pant P-184, Pant P-186, Pant P-195, Pant P-200, Pant P-2009-3, Pant P-207, Pant P-215, Pant P-217, Pant P- } \\
\text { 222, Pant P-223, Pant P-247, Pant P-250, Pant P-347, Pant P-365, Pant P-367, Pant P-375, Pant P-38, Pant P-397, } \\
\text { Pant P-399, Pant P-402, Pant P-42, PH-1, RFP 11-09, RFP 2009-2, RFP 2010-11, RFP 2010-3, RFP 2011-1, RFP } \\
\text { 72, RFPG 112, RFPG 114, SKUAP-8, SPC101, VL-201, VL-202, VL-55, VL-56, VL-58, V L-66, VL-67 }\end{array}$ & 126 \\
\hline & & Vestigial:3 & None & 0 \\
\hline 6. & $\begin{array}{l}\text { Foliage: Waxy } \\
\text { bloom }\end{array}$ & Absent:1 & $\begin{array}{l}\text { 20/1062, Ambika, DDR-36, DDR-4, DMR-7, HFP 0110, HFP 0130, HFP 0145, HFP 0149, HFP 1010, HFP 1024, } \\
\text { HFP 1104, HFP 1120, HFP 1130, HFP 1140, HFP 1201, HFP 1306, HFP 1425, HFP 1428, FP 1463, HFP 9106, } \\
\text { HFP 920, HFP 9426, HFP 9907A, HUDP 1301, HUDP 1711, IP2K 83-1, IPF 11-13, IPF 11-15, IPF 17-18, IPF } \\
\text { 17-19, IPF 5-19, IPFD 12-8, IPFD 13-14, IPFD 13-2, IPFD 17-2, IPFD 2014-11, KPF 1024, KPF 115, KPF 14-35, } \\
\text { KPF 14-36, KPF 14-64, KPMR 745, KPMR 851, KPMR 910, KPMR 935, NDP 11-101, NDP 11-102, NDP 14- } \\
\text { 11, NDPD 2017-06, NDPT 2017-04, NGSN-13, NGSN-14,NGSN-3, NGSN-4,NGSN-5, NGSN-8, NGSN-9, Pant }\end{array}$ & 92 \\
\hline
\end{tabular}




\begin{tabular}{|c|c|c|c|c|}
\hline & & & $\begin{array}{l}\text { P-125, Pant P-180, Pant P-184, Pant P-186, Pant P-195, Pant P-200, Pant P-2009-3, Pant P-207, Pant P-217, Pant } \\
\text { P-222, Pant P-223, Pant P-247, Pant P-347, Pant P-375, Pant P-38, Pant P-397, Pant P-399, Pant P-402, Pant P-42, } \\
\text { PH-1, RFP 11-09, RFP 2009-2, RFP 2010-11, RFP 2010-3, RFP 2011-1, RFP 72, RFPG 112, SKUAP-8, VL-201, } \\
\text { VL-202, VL-56, VL-58, VL-66, VL-67 }\end{array}$ & \\
\hline & & Present:9 & $\begin{array}{l}\text { Aman, EC 398601, EC 412882, HFP 1036, HFP 1107, HFP 1125,HFP 1129, HFP 1132,HFP 1137, HFP 1302, } \\
\text { HFP 1307, HFP 1314, HFP 1315, HFP 1405, HFP 2008, HFP 530B, HFP 9907B, HUDP 1302, HUDP 1715, } \\
\text { HUPT 1701, IPFD 12-2, IPFD 17-6, KFP 12-4, KPMR 928, NGSN-10, NGSN-11, NGSN-6, Pant P-215, Pant P- } \\
\text { 250, Pant P-365,Pant P-367, RFPG 114, SPC101, VL-55 }\end{array}$ & 34 \\
\hline \multirow[t]{2}{*}{7.} & \multirow[t]{2}{*}{ Leaf: Axil colour } & Green:1 & $\begin{array}{l}\text { 20/1062, Aman, Ambika, DDR-36, DMR-7, HFP 0110, HFP 0130, HFP 0145, HFP 0149, HFP 1010, HFP 1024, } \\
\text { HFP 1036, HFP 1104, HFP 1107, HFP 1120, HFP 1125, HFP 1129, HFP 1130, HFP 1132, HFP 1137, HFP 1140, } \\
\text { HFP 1201, HFP 1302, HFP 1306, HFP 1307, HFP 1314, HFP 1315, HFP 1405, HFP 1425, HFP 1428, HFP 1463, } \\
\text { HFP 2008, HFP 530B, HFP 9106, HFP 920, HFP 9426, HFP 9907A, HFP 9907B , HUDP 1301, HUDP 1302, } \\
\text { HUDP 1711, HUDP 1715, HUPT 1701, IPF 11-13, IPF 17-18, IPF 17-19, IPF 5-19, IPFD 12-2, IPFD 12-8, IPFD } \\
\text { 13-14, IPFD 13-2, IPFD 17-2, IPFD 17-6, IPFD 2014-11, KFP 12-4, KPF 1024, KPF 115, KPF 14-35, KPF 14-36, } \\
\text { KPF 14-64, KPMR 745, KPMR 851, KPMR 910, KPMR 928, KPMR 935, NDP 11-101, NDP 11-102, NDP 14- } \\
\text { 11, NDPD 2017-06, NDPT 2017-04, NGSN-14, NGSN-3, NGSN-4, NGSN-5, NGSN-8, NGSN-9, Pant P-125, } \\
\text { Pant P-180, Pant P-184, Pant P-186, Pant P-195, Pant P-200, Pant P-207, Pant P-215, Pant P-217, Pant P-222, Pant } \\
\text { P-223, Pant P-247, Pant P-250, Pant P-347, Pant P-365, Pant P-367, Pant P-375, Pant P-38, Pant P-397, Pant P- } \\
\text { 399, Pant P-402, Pant P-42, PH-1, RFP 11-09, RFP 2010-11, RFP 2010-3, RFP 2011-1, RFP 72, RFPG 112, } \\
\text { RFPG 114, SKUAP-8, SPC101, VL-201, VL-202, VL-55, VL-56, VL-58, V L-66, VL-67 }\end{array}$ & 115 \\
\hline & & Purple:2 & $\begin{array}{l}\text { DDR-4, EC 398601, EC 412882, IP2K 83-1, IPF 11-15, NGSN-10, NGSN-11, NGSN-13, NGSN-6, Pant P-2009- } \\
\text { 3, RFP 2009-2 }\end{array}$ & 11 \\
\hline \multirow[t]{4}{*}{8.} & \multirow{4}{*}{$\begin{array}{l}\text { Time of flowering } \\
\text { (Days to } 50 \% \\
\text { flowering) }\end{array}$} & $\begin{array}{l}\text { Extra early } \\
(<40): 1\end{array}$ & None & 0 \\
\hline & & $\begin{array}{c}\text { Early } \\
(40-50): 2\end{array}$ & None & 0 \\
\hline & & $\begin{array}{l}\text { Medium } \\
(51-70): 3\end{array}$ & $\begin{array}{l}\text { Ambika, DDR-4, HFP 1104, HFP 9907A, HUDP 1301,HUDP 1302, IPF 5-19, NGSN-10, NGSN-11, NGSN-13, } \\
\text { Pant P-399, Pant P-402, PH-1, RFP 72 }\end{array}$ & 14 \\
\hline & & Late $(>70): 4$ & $\begin{array}{l}\text { 20/1062, Aman, DDR-36, DMR-7, EC 398601, EC 412882, HFP 0110, HFP 0130, HFP 0145, HFP 0149, HFP } \\
\text { 1010, HFP 1024, HFP 1036, HFP 1107, HFP 1120, HFP 1125, HFP 1129, HFP 1130, HFP 1132, HFP 1137, HFP } \\
\text { 1140, HFP 1201, HFP 1302, HFP 1306, HFP 1307, HFP 1314, HFP 1315, HFP 1405, HFP 1425, HFP 1428, HFP } \\
\text { 1463, HFP 2008, HFP 530B, HFP 9106, HFP 920, HFP 9426, HFP 9907B , HUDP 1711, HUDP 1715, HUPT } \\
\text { 1701, IP2K 83-1, IPF 11-13, IPF 11-15, IPF 17-18, IPF 17-19, IPFD 12-2, IPFD 12-8, IPFD 13-14, IPFD 13-2, } \\
\text { IPFD 17-2, IPFD 17-6, IPFD 2014-11, KFP 12-4, KPF 1024, KPF 115, KPF 14-35, KPF 14-36, KPF 14-64, } \\
\text { KPMR 745, KPMR 851, KPMR 910, KPMR 928, KPMR 935, NDP 11-101, NDP 11-102, NDP 14-11, NDPD } \\
\text { 2017-06, NDPT 2017-04, NGSN-14, NGSN-3, NGSN-4, NGSN-5, NGSN-6, NGSN-8, NGSN-9, Pant P-125, Pant } \\
\text { P-180, Pant P-184, Pant P-186, Pant P-195, Pant P-200, Pant P-2009-3, Pant P-207, Pant P-215, Pant P-217, Pant } \\
\text { P-222, Pant P-223, Pant P-247, Pant P-250, Pant P-347, Pant P-365, Pant P-367, Pant P-375, Pant P-38, Pant P- } \\
\text { 397, Pant P-42, RFP 11-09, RFP 2009-2, RFP 2010-11, RFP 2010-3, RFP 2011-1, RFPG 112, RFPG 114, }\end{array}$ & 112 \\
\hline
\end{tabular}




\begin{tabular}{|c|c|c|c|c|}
\hline & & & SKUAP-8, SPC101, VL-201, VL-202, VL-55, VL-56, VL-58, VL-66, VL-67 & \\
\hline \multirow[t]{5}{*}{9.} & \multirow[t]{5}{*}{$\begin{array}{l}\text { Flower: Standard } \\
\text { petal colour }\end{array}$} & White: 1 & $\begin{array}{l}\text { 20/1062, Aman, Ambika, DDR-36, DMR-7, HFP 0110, HFP 0130, HFP 0145, HFP 0149, HFP 1010, HFP 1024, } \\
\text { HFP 1036, HFP 1104, HFP 1107, HFP 1120, HFP 1125, HFP 1129, HFP 1130, HFP 1132, HFP 1137, HFP 1140, } \\
\text { HFP 1201, HFP 1302, HFP 1306, HFP 1307, HFP 1314, HFP 1315, HFP 1405, HFP 1425, HFP 1428, HFP 1463, } \\
\text { HFP 2008, HFP 530B, HFP 9106, HFP 920, HFP 9426, HFP 9907A, HFP 9907B , HUDP 1301, HUDP 1302, } \\
\text { HUDP 1711, HUDP 1715, HUPT 1701,IPF 11-13, IPF 17-18,IPF 17-19,IPF 5-19,IPFD 12-2,IPFD 12-8, IPFD 13- } \\
\text { 14,IPFD 13-2,IPFD 17-2,IPFD 17-6, IPFD 2014-11, KFP 12-4, KPF 1024, KPF 115, KPF 14-35, KPF 14-36, KPF } \\
\text { 14-64, KPMR 745, KPMR 851, KPMR 910, KPMR 928, KPMR 935, NDP 11-101, NDP 11-102, NDP 14-11, } \\
\text { NDPD 2017-06, NDPT 2017-04, NGSN-14, NGSN-3, NGSN-4, NGSN-5, NGSN-8, NGSN-9, Pant P-125, Pant } \\
\text { P-180, Pant P-184, Pant P-186, Pant P-195, Pant P-200, Pant P-207, Pant P-215, Pant P-217, Pant P-222, Pant P- } \\
\text { 223, Pant P-247, Pant P-250, Pant P-347, Pant P-365, Pant P-367, Pant P-375, Pant P-38, Pant P-397, Pant P-399, } \\
\text { Pant P-402, Pant P-42, PH-1, RFP 11-09, RFP 2010-11, RFP 2010-3, RFP 2011-1, RFP 72, RFPG 112, RFPG } \\
\text { 114, SKUAP-8, SPC101, VL-201, VL-202, VL-55, VL-56, VL-58, VL-66, VL-67 }\end{array}$ & 115 \\
\hline & & Blue:2 & None & 0 \\
\hline & & Pink:3 & None & 0 \\
\hline & & Red:4 & None & 0 \\
\hline & & Purple:5 & $\begin{array}{l}\text { DDR-4, EC 398601, EC 412882, IP2K 83-1, IPF 11-15, NGSN-10, NGSN-11, NGSN-13, NGSN-6, Pant P-2009- } \\
3 \text {, RFP 2009-2 }\end{array}$ & 11 \\
\hline \multirow[t]{3}{*}{10.} & \multirow{3}{*}{$\begin{array}{c}\text { Pod: Number / } \\
\text { Axil }\end{array}$} & Single: 1 & DDR-4, HFP 0130, IP2K 83-1, KPMR 745, NDP 11-101, NGSN-3 & 6 \\
\hline & & Double:2 & $\begin{array}{l}\text { 20/1062, Aman, Ambika, DDR-36, DMR-7, EC 398601, EC 412882, HFP 0110, HFP 0145, HFP 0149, HFP 1010, } \\
\text { HFP 1024, HFP 1036, HFP 1104, HFP 1107, HFP 1120, HFP 1125, HFP 1129, HFP 1130, HFP 1132, HFP 1137, } \\
\text { HFP 1140, HFP 1201, HFP 1302, HFP 1306, HFP 1307, HFP 1314, HFP 1315, HFP 1405, HFP 1425, HFP 1428, } \\
\text { HFP 1463, HFP 2008, HFP 530B, HFP 9106, HFP 920, HFP 9426, HFP 9907A, HFP 9907B , HUDP 1301, } \\
\text { HUDP 1302, HUDP 1711, HUDP 1715, HUPT 1701, IPF 11-13, IPF 11-15, IPF 17-18, IPF 17-19, IPF 5-19, IPFD } \\
\text { 12-2, IPFD 12-8, IPFD 13-14, IPFD 13-2, IPFD 17-2, IPFD 17-6, IPFD 2014-11, KFP 12-4, KPF 1024, KPF 115, } \\
\text { KPF 14-35, KPF 14-36, KPF 14-64, KPMR 745, KPMR 851, KPMR 910, KPMR 928, KPMR 935, NDP 11-102, } \\
\text { NDP 14-11, NDPD 2017-06, NDPT 2017-04, NGSN-10, NGSN-11, NGSN-13, NGSN-14, NGSN-4, NGSN-5, } \\
\text { NGSN-6, NGSN-8, NGSN-9, Pant P-125, Pant P-180, Pant P-184, Pant P-186, Pant P-195, Pant P-200, Pant P- } \\
\text { 2009-3, Pant P-207, Pant P-215, Pant P-21, Pant P-222, Pant P-223, Pant P-247, Pant P-250, Pant P-347, Pant P- } \\
\text { 365, Pant P-367, Pant P-375, Pant P-38, Pant P-397, Pant P-399, Pant P-402, Pant P-42, PH-1, RFP 11-09, RFP } \\
\text { 2009-2, RFP 2010-11, RFP 2010-3, RFP 2011-1, RFP 72, RFPG 112, RFPG 114, SKUAP-8, SPC101, VL201, } \\
\text { VL-202, VL-55, VL-56, VL-58, VL-66, VL-67 }\end{array}$ & 120 \\
\hline & & Multiple:3 & None & 0 \\
\hline \multirow[t]{2}{*}{11.} & \multirow[t]{2}{*}{ Pod: Curvature } & Absent:1 & EC 412882 & 1 \\
\hline & & Weak:3 & $\begin{array}{l}\text { 20/1062, DDR-36, DDR-4, DMR-7, EC } 398601 \text {, HFP } 0145 \text {, HFP 0149, HFP 1010, HFP 1036, HFP 1104, HFP } \\
\text { 1120, HFP 1125, HFP 1129, HFP 1130, HFP 1132, HFP 1137, HFP 1140, HFP 1302, HFP 1306, HFP 1307, HFP } \\
\text { 530B,HFP 9106, HFP 920, HUDP 1301, HUDP 1302, HUPT 1701,IP2K 83-1,IPF 11-13,IPF 11-15, IPF 5-19, IPFD } \\
\text { 12-2, IPFD 12-8, IPFD 13-14, IPFD 13-2, IPFD 17-2, KPF 1024, KPF 115, KPF 14-35, KPF 14-36, KPF 14-64, } \\
\text { KPMR 745, KPMR 851, KPMR 910, KPMR 935, NDP 11-102, NDPT 2017-04, NGSN-11, NGSN-14, NGSN-3, }\end{array}$ & 84 \\
\hline
\end{tabular}




\begin{tabular}{|c|c|c|c|c|}
\hline & & & $\begin{array}{l}\text { NGSN-5, NGSN-6, NGSN-8, NGSN-9, Pant P-125, Pant P-180, Pant P-184,Pant P-186, Pant P-195, Pant P-200, } \\
\text { Pant P-2009-3, Pant P-207, Pant P-215, Pant P-247, Pant P-347, Pant P-367, Pant P-375, Pant P-38, Pant P-397, } \\
\text { Pant P-399, Pant P-402, PH-1, RFP 11-09, RFP 2009-2, RFP 2010-11, RFP 2010-3, RFP 2011-1, RFP 72, RFPG } \\
\text { 114, SKUAP-8, VL-202, VL-55, VL-56, VL-58, VL-66 }\end{array}$ & \\
\hline & & Medium:5 & $\begin{array}{l}\text { Aman, Ambika, HFP 0110, HFP 0130, HFP 1024, HFP 1107, HFP 1201, HFP 1314, HFP 1315, HFP 1405, HFP } \\
\text { 1425, HFP 1428, HFP 1463, HFP 2008, HFP 9426, HFP 9907A, HFP 9907B, HUDP 1711, HUDP 1715, IPF 17- } \\
\text { 18, IPF 17-19, IPFD 17-6, IPFD 2014-11, KFP 12-4, KPMR 928, NDP 11-101, NDP 14-11, NDPD 2017-06, } \\
\text { NGSN-13, NGSN-4, Pant P-217, Pant P-222, Pant P-223, Pant P-250, Pant P-365, Pant P-42, RFPG 112, SPC101, } \\
\text { VL-201, VL-67 }\end{array}$ & 40 \\
\hline & & Strong:7 & NGSN-10 & 1 \\
\hline \multirow[t]{2}{*}{12.} & \multirow[t]{2}{*}{$\begin{array}{c}\text { Pod: Shape of } \\
\text { distal part }\end{array}$} & Pointed:1 & $\begin{array}{l}\text { 20/1062, Aman, Ambika, DDR-36, DDR-4, EC 398601, EC 412882, HFP 0130, HFP 0145, HFP 1104, HFP 1107, } \\
\text { HFP 1130, HFP 1140, HFP 1201, HFP 1306, HFP 1314, HFP 1425, HFP 1428, HFP 2008, HFP 530B, HFP 9106, } \\
\text { HUDP 1301, HUDP 1302, HUDP 1711, HUDP 1715, IPF 11-13, IPF 5-19, IPFD 13-2, PFD 17-2, IPFD 2014-11, } \\
\text { KFP 12-4, KPF 1024, KPF 14-35, KPF 14-64, KPMR 745, KPMR 851, KPMR 928, NDP 11-101, NDP 14-11, } \\
\text { NGSN-10, NGSN-4, NGSN-5, NGSN-9, Pant P-186, Pant P-125, Pant P-180, Pant P-184, Pant P-207, Pant P-215, } \\
\text { Pant P-217, Pant P-222, Pant P-223, Pant P-247, Pant P-250, Pant P-365, Pant P-367, Pant P-397, Pant P-42, RFP } \\
\text { 2009-2, RFP 72, SPC101, VL-201, VL-56 }\end{array}$ & 63 \\
\hline & & Blunt:9 & $\begin{array}{l}\text { DMR-7, HFP 0110, HFP 0149, HFP 1010, HFP 1024, HFP 1036, HFP 1120, HFP 1125, HFP 1129, HFP 1132, } \\
\text { HFP 1137, HFP 1302, HFP 1307, HFP 1315, HFP 1405, HFP 1463, HFP 920, HFP 9426, HFP 9907A, HFP } \\
\text { 9907B, HUPT 1701, IP2K 83-1, IPF 11-15, IPF 17-18,IPF 17-19, IPFD 12-2, IPFD 12-8, IPFD 17-6, KPMR 910, } \\
\text { IPFD 13-14, KPF 115, KPF 14-36, KPMR 935, NDP 11-102, NDPD 2017-06, NDPT 2017-04, NGSN-11, NGSN- } \\
\text { 13, NGSN-14, NGSN-3, NGSN-6, NGSN-8, Pant P-195, Pant P-200, Pant P-2009-3, Pant P-347, Pant P-375, Pant } \\
\text { P-38, Pant P-399, Pant P-402, PH-1, RFP 11-09, RFP 2010-11, RFP 2010-3, RFP 2011-1, RFPG 112, RFPG 114, } \\
\text { SKUAP-8, VL-202, VL-55, VL-58, VL-66, VL-67 }\end{array}$ & 63 \\
\hline \multirow[t]{2}{*}{13.} & \multirow[t]{2}{*}{$\begin{array}{l}\text { Pod: Intensity of } \\
\text { green colour }\end{array}$} & $\begin{array}{l}\text { Light } \\
\text { green:3 }\end{array}$ & HFP 0130, HFP 1306, Pant P-200 & 3 \\
\hline & & Green:5 & $\begin{array}{l}\text { 20/1062, Aman, Ambika, DDR-36, DDR-4, DMR-7, EC 398601, EC 412882, HFP 0110, HFP 0145, HFP } 0149 \\
\text { HFP 1010, HFP 1024, HFP 1036, HFP 1104, HFP 1107, HFP 1120, HFP 1125, HFP 1129, HFP 1130, HFP 1132, } \\
\text { HFP 1137, HFP 1140, HFP 1201, HFP 1302, HFP 1307, HFP 1314, HFP 1315, HFP 1405, HFP 1425, HFP 1428, } \\
\text { HFP 1463, HFP 2008, HFP 530B, HFP 9106, HFP 920, HFP 9426, HFP 9907A, HFP 9907B, HUDP 1301, HUDP } \\
\text { 1302, HUDP 1711, HUDP 1715, HUPT 1701, IP2K 83-1, IPF 11-13, IPF 11-15, IPF 17-18, IPF 17-19, IPF 5-19, } \\
\text { IPFD 12-2, IPFD 12-8, IPFD 13-14, IPFD 13-2, IPFD 17-2, IPFD 17-6, IPFD 2014-11, KFP 12-4, KPF 1024, KPF } \\
\text { 115, KPF 14-35, KPF 14-36, KPF 14-64, KPMR 745, KPMR 851, KPMR 910, KPMR 928, KPMR 935, NDP 11- } \\
\text { 101, NDP 11-102, NDP 14-11, NDPD 2017-06, NDPT 2017-04, NGSN-10, NGSN-11, NGSN-13, NGSN-14, } \\
\text { NGSN-3, NGSN-4, NGSN-5, NGSN-6, NGSN-8, NGSN-9, Pant P-125, Pant P-180, Pant P-184, Pant P-186, Pant } \\
\text { P-195, Pant P-2009-3, Pant P-207, Pant P-215, Pant P-217, Pant P-222, Pant P-223, Pant P-247, Pant P-250, Pant } \\
\text { P-347, Pant P-365, Pant P-367, Pant P-375, Pant P-38, Pant P-397, Pant P-399, Pant P-402, Pant P-42, PH-1, RFP } \\
\text { 11-09, RFP 2009-2, RFP 2010-11, RFP 2010-3, RFP 2011-1, RFP 72, RFPG 112, RFPG 114, SKUAP-8, SPC101, } \\
\text { VL-201, VL-202, VL-55, VL-56, VL-58, VL-66, VL-67 }\end{array}$ & 123 \\
\hline
\end{tabular}




\begin{tabular}{|c|c|c|c|c|}
\hline & & Dark green:7 & None & 0 \\
\hline \multirow[t]{3}{*}{14.} & \multirow[t]{3}{*}{ Plant height } & $\begin{array}{c}\text { Short } \\
(<60 \mathrm{~cm}): 3\end{array}$ & None & 0 \\
\hline & & $\begin{array}{l}\text { Medium } \\
(60-80 \mathrm{~cm}): 5\end{array}$ & HFP 1306, KPMR 928, Pant P-207, Pant P-223, VL-201 & 5 \\
\hline & & $\begin{array}{l}\text { Long } \\
(>80 \mathrm{~cm}): 7\end{array}$ & $\begin{array}{l}\text { 20/1062, Aman, Ambika, DDR-36, DDR-4, DMR-7, EC 398601, EC 412882, HFP 0110, HFP 0130, HFP 0145, } \\
\text { HFP 0149, HFP 1010, HFP 1024, HFP 1036, HFP 1104, HFP 1107, HFP 1120, HFP 1125, HFP 1129, HFP 1130, } \\
\text { HFP 1132, HFP 1137, HFP 1140, HFP 1201, HFP 1302, HFP 1307, HFP 1314, HFP 1315, HFP 1405, HFP 1425, } \\
\text { HFP 1428, HFP 1463, HFP 2008, HFP 530B, HFP 9106, HFP 920, HFP 9426, HFP 9907A, HFP 9907B, HUDP } \\
\text { 1301, HUDP 1302, HUDP 1711, HUDP 1715, HUPT 1701, IP2K 83-1, IPF 11-13, IPF 11-15, IPF 17-18, IPF 17- } \\
\text { 19, IPF 5-19, IPFD 12-2, IPFD 12-8, IPFD 13-14, IPFD 13-2, IPFD 17-2, IPFD 17-6, IPFD 2014-11, KFP 12-4, } \\
\text { KPF 1024, KPF 115, KPF 14-35, KPF 14-36, KPF 14-64, KPMR 745, KPMR 851, KPMR 910, KPMR 935, NDP } \\
\text { 11-101, NDP 11-102, NDP 14-11, NDPD 2017-06, NDPT2017-04, NGSN-10, NGSN-11, NGSN-13, NGSN-14, } \\
\text { NGSN-3, NGSN-4, NGSN-5, NGSN-6, NGSN-8, NGSN-9, Pant P-125, Pant P-180, Pant P-184, Pant P-186, Pant } \\
\text { P-195, Pant P-200, Pant P-2009-3, Pant P-215, Pant P-217, Pant P-222, Pant P-247, Pant P-250, Pant P-347, Pant } \\
\text { P-365, Pant P-367, Pant P-375, Pant P-38, Pant P-397, Pant P-399, Pant P-402, Pant P-42, PH-1, RFP 11-09, RFP } \\
\text { 2009-2, RFP 2010-11, RFP 2010-3, RFP 2011-1, RFP 72, RFPG 112, RFPG 114, SKUAP-8, SPC101, VL-202, } \\
\text { VL-55, VL-56, VL-58, VL-66, VL-67 }\end{array}$ & 121 \\
\hline \multirow[t]{3}{*}{15.} & \multirow[t]{3}{*}{ Seed: Shape } & Spherical: 1 & $\begin{array}{l}\text { 20/1062, Aman, Ambika, DDR-36, DDR-4, DMR-7, HFP 0130, HFP 0145, HFP 0149, HFP 1024, HFP 1036, } \\
\text { HFP 1104, HFP 1129, HFP 1130, HFP 1132, HFP 1140, HFP 1201, HFP 1302, HFP 1315, HFP 1425, HFP 920, } \\
\text { HFP 9426, HUDP 1302, HUDP 1711, HUDP 1715, HUPT 1701, IP2K 83-1, IPF 11-13, IPF 17-18, IPF 17-19, IPF } \\
\text { 5-19, IPFD 12-2, IPFD 12-8, IPFD 13-14, IPFD 13-2, IPFD 2014-11, KFP 12-4, KPF 1024, KPF 14-35, KPMR } \\
\text { 745, KPMR 851, KPMR 910, KPMR 928, KPMR 935, NDP 11-102, NDP 14-11, NDPD 2017-06, NDPT2017-04, } \\
\text { NGSN-10, NGSN-13, NGSN-14, NGSN-3, NGSN-4, NGSN-5, NGSN-6, NGSN-8, Pant P-125, Pant P-180, Pant } \\
\text { P-184, Pant P-186, Pant P-195, Pant P-200, Pant P-207, Pant P-217, Pant P-222, Pant P-250, Pant P-365, Pant P- } \\
\text { 367, Pant P-38, Pant P-397, Pant P-399, Pant P-402, Pant P-42, PH-1, RFP 11-09, RFP 2010-11, RFP 2010-3, RFP } \\
\text { 2011-1, RFP 72, RFPG 112, RFPG 114, SKUAP-8, SPC101, VL-201, VL-202, VL-55, VL-56, VL-58, VL-66, } \\
\text { VL-67 }\end{array}$ & 91 \\
\hline & & Cylindrical:2 & $\begin{array}{l}\text { HFP 1107, HFP 1120, HFP 1125, HFP 1307, HFP 1314, HFP 1428, HFP 9106, HFP 9907A, HFP 9907B, HUDP } \\
\text { 1301, IPFD 17-2, KPF 115, KPF 14-36, KPF 14-64, NDP 11-101, Pant P-375 }\end{array}$ & 16 \\
\hline & & Dimpled:3 & $\begin{array}{l}\text { EC 398601, EC 412882, HFP 0110, HFP 1010, HFP 1137, HFP 1306, HFP 1405, HFP 1463, HFP 2008, HFP } \\
\text { 530B, IPF 11-15, NGSN-11, NGSN-9, Pant P-2009-3, Pant P-215, Pant P-223, Pant P-247, Pant P-347,RFP 2009- } \\
2\end{array}$ & 19 \\
\hline 16. & Seed: Surface & Smooth:1 & $\begin{array}{l}\text { 20/1062, Aman, DMR-7, HFP 0130, HFP 0145, HFP 0149, HFP 1132, HFP 1302, HFP 1307, HFP 9426, HUDP } \\
\text { 1715, IPF 17-18, IPF 17-19, IPF 5-19, IPFD 12-2, IPFD 13-2, IPFD 17-6, KPF 1024, KPF 14-35, KPMR 745, } \\
\text { KPMR 851, KPMR 910, KPMR 935, NDP 14-11, NDPD 2017-06, NDPT 2017-04, NGSN-3, NGSN-4, NGSN-5, } \\
\text { Pant P-184, Pant P-186, Pant P-200, Pant P-207, Pant P-217, Pant P-250, Pant P-367, Pant P-38, Pant P-399, Pant } \\
\text { P-402, Pant P-42, RFP 2010-3, RFP 2011-1, RFP 72, RFPG 112, SKUAP-8, SPC101, VL-201, VL-202, VL-55, } \\
\text { VL-58, VL-66, VL-67 }\end{array}$ & 52 \\
\hline
\end{tabular}




\begin{tabular}{|c|c|c|c|c|}
\hline & & Wrinkled:2 & $\begin{array}{l}\text { Ambika, DDR-36, DDR-4, EC 398601, EC 412882, HFP 0110, HFP 1010, HFP 1024, HFP 1036, HFP 1104, HFP } \\
\text { 1107, HFP 1120, HFP 1125, HFP 1129, HFP 1130, HFP 1137, HFP 1140, HFP 1201, HFP 1306, HFP 1314, HFP } \\
\text { 1315, HFP 1405,HFP 1425, HFP 1428, HFP 1463, HFP 2008, HFP 530B, HFP 9106, HFP 920, HFP 9907A, HFP } \\
\text { 9907B, HUDP 1301, HUDP 1302, HUDP 1711, HUPT 1701, IP2K 83-1, IPF 11-13, IPF 11-15, IPFD 12-8, IPFD } \\
\text { 13-14, IPFD 17-2, IPFD 2014-11,KFP 12-4, KPF 115, KPF 14-36, KPF 14-64, KPMR 928, NDP 11-101, NDP } \\
\text { 11-102, NGSN-10, NGSN-11, NGSN-13, NGSN-14, NGSN-6, NGSN-8, NGSN-9, Pant P-125, Pant P-180, Pant } \\
\text { P-195, Pant P-2009-3, Pant P-215, Pant P-222, Pant P-223, Pant P-247, Pant P-347, Pant P-365, Pant P-375, Pant } \\
\text { P-397, PH-1, RFP 11-09, RFP 2009-2, RFP 2010-11, RFPG 114, VL-56 }\end{array}$ & 74 \\
\hline \multirow[t]{3}{*}{17.} & \multirow{3}{*}{$\begin{array}{l}\text { Seed: Cotyledon } \\
\text { colour }\end{array}$} & Creamy:3 & None & 0 \\
\hline & & Green:5 & $\begin{array}{l}\text { DDR-4, EC 398601, HFP 1104, HFP 1107, HFP 1120, HFP 1129, HFP 1130, HFP 1132, HFP 1137, HFP 1140, } \\
\text { HFP 1201, HFP 1314, HFP 1315, HFP 1463, HFP 2008, HFP 9426, HFP 9907A, HFP 9907B, IP2K 83-1, IPF 17- } \\
\text { 18, IPFD 17-2, KPF 115, KPF 14-36, NGSN-10, NGSN-13, NGSN-14, NGSN-6, Pant P-215, PH-1,RFP 11-09, } \\
\text { RFP 2009-2 }\end{array}$ & 31 \\
\hline & & Yellow:7 & $\begin{array}{l}\text { 20/1062, Aman, Ambika, DDR-36, DMR-7, EC 412882, HFP 0110, HFP 0130, HFP 0145, HFP 0149, HFP 1010, } \\
\text { HFP 1024, HFP 1036, HFP 1125, HFP 1302, HFP 1306, HFP 1307, HFP 1405, HFP 1425, HFP 1428, HFP 530B, } \\
\text { HFP 9106, HFP 920, HUDP 1301, HUDP 1302, HUDP 1711, HUDP 1715, HUPT 1701, IPF 11-13, IPF 11-15, } \\
\text { IPF 17-19, IPF 5-19, IPFD 12-2, IPFD 12-8, IPFD 13-14, IPFD 13-2, IPFD 17-6, IPFD 2014-11, KFP 12-4, KPF } \\
\text { 1024, KPF 14-35, KPF 14-64,KPMR 745,KPMR 851,KPMR 910, KPMR 928,KPMR 935, NDP 11-101, NDP 11- } \\
\text { 102, NDP 14-11, NDPD 2017-06, NDPT 2017-04, NGSN-11, NGSN-3, NGSN-4, NGSN-5, NGSN-8, NGSN-9, } \\
\text { Pant P-125, Pant P-180, Pant P-184, Pant P-186, Pant P-195, Pant P-200, Pant P-2009-3, Pant P-207, Pant P-217, } \\
\text { Pant P-222, Pant P-223, Pant P-247, Pant P-250, Pant P-347, Pant P-365, Pant P-367, Pant P-375, Pant P-38, Pant } \\
\text { P-397, Pant P-399, Pant P-402, Pant P-42, RFP 2010-11, RFP 2010-3, RFP 2011-1, RFP 72, RFPG 112, RFPG } \\
\text { 114, SKUAP-8, SPC101, VL-201, VL-202, VL-55, VL-56, VL-58, VL-66, VL-67 }\end{array}$ & 95 \\
\hline \multirow[t]{3}{*}{18.} & \multirow[t]{3}{*}{$\begin{array}{l}\text { Seed: } 100 \text {-seed } \\
\text { weight }(\mathrm{g})\end{array}$} & $\begin{array}{c}\text { Small } \\
(<15 \mathrm{~g}): 3\end{array}$ & $\begin{array}{l}\text { 20/1062, Aman, Ambika, EC 412882, HFP 0110, HFP 0130, HFP 0145, HFP 0149, HFP 9106, HFP 9907A, } \\
\text { HUDP 1301, HUDP 1302, HUDP 1711, HUDP 1715, HUPT 1701, IPF 5-19, KPF 115, KPMR 745, KPMR 851, } \\
\text { KPMR 928, NDP 11-101, NDP 11-102, NDPD 2017-06, NGSN-10, NGSN-13, NGSN-14, NGSN-3, NGSN-4, } \\
\text { NGSN-5, NGSN-6, NGSN-8, NGSN-9, Pant P-125, Pant P-180, Pant P-186, PH-1, RFP 2009-2, RFP 72, VL-55, } \\
\text { VL-56, VL-58 }\end{array}$ & 41 \\
\hline & & $\begin{array}{l}\text { Medium } \\
(15-20 \mathrm{~g}): 5\end{array}$ & $\begin{array}{l}\text { DDR-36, DDR-4, DMR-7, EC 398601, HFP 1010, HFP 1024, HFP 1036, HFP 1104, HFP 1107, HFP 1120, HFP } \\
\text { 1129, HFP 1130, HFP 1132, HFP 1137, HFP 1140, HFP 1201, HFP 1302, HFP 1306, HFP 1307, HFP 1314, HFP } \\
\text { 1315, HFP 1405, HFP 1425, HFP 1428, HFP 1463, HFP 2008, HFP 530B, HFP 920, HFP 9426, HFP 9907B, } \\
\text { IP2K 83-1, IPF 11-13, IPF 11-15, IPF 17-18, IPF 17-19, IPFD 12-2, IPFD 12-8, IPFD 13-14, IPFD 13-2, IPFD 17- } \\
\text { 2, IPFD 17-6, IPFD 2014-11, KFP 12-4, KPF 1024, KPF 14-36, KPF 14-64, KPMR 910, KPMR 935, NDP 14-11, } \\
\text { NDPT 2017-04, NGSN-11, Pant P-184, Pant P-195, Pant P-200, Pant P-2009-3, Pant P-207, Pant P-215, Pant P- } \\
\text { 217, Pant P-222, Pant P-223, Pant P-247, Pant P-250, Pant P-347, Pant P-365, Pant P-367, Pant P-375, Pant P-38, } \\
\text { Pant P-397, Pant P-399, Pant P-402, Pant P-42, RFP 11-09, RFP 2010-11, RFP 2010-3, RFP 2011-1, RFPG 112, } \\
\text { SKUAP-8, SPC101, VL-201, VL-202, VL-66, VL-67 }\end{array}$ & 82 \\
\hline & & $\begin{array}{c}\text { Large } \\
(>20 \mathrm{~g}): 7\end{array}$ & HFP 1125, KPF 14-35, RFPG 114 & 3 \\
\hline
\end{tabular}




\begin{tabular}{|c|c|c|c|c|}
\hline \multirow[t]{2}{*}{19.} & \multirow[t]{2}{*}{$\begin{array}{l}\text { Seed: Testa } \\
\text { mottling }\end{array}$} & Absent: 1 & $\begin{array}{l}\text { 20/1062, Aman, Ambika, DDR-36, DDR-4, DMR-7, EC 398601, EC 412882, HFP 0110, HFP 0130, HFP 0145, } \\
\text { HFP 0149, HFP 1010, HFP 1024, HFP 1036, HFP 1104, HFP 1107, HFP 1120, HFP 1125, HFP 1129, HFP 1130, } \\
\text { HFP 1132, HFP 1137, HFP 1140, HFP 1201, HFP 1302, HFP 1306, HFP 1307, HFP 1314, HFP 1315, HFP 1405, } \\
\text { HFP 1425, HFP 1428, HFP 1463, HFP 2008, HFP 530B, HFP 9106, HFP 920, HFP 9426, HFP 9907A, HFP } \\
\text { 9907B, HUDP 1301, HUDP 1302, HUDP 1711, HUDP 1715, HUPT 1701, IP2K 83-1, IPF 11-13, IPF 11-15, IPF } \\
\text { 17-18, IPF 17-19, IPF 5-19, IPFD 12-2, IPFD 12-8, IPFD 13-14, IPFD 13-2, IPFD 17-2, IPFD 17-6, IPFD 2014- } \\
\text { 11, KFP 12-4, KPF 1024, KPF 115, KPF 14-35, KPF 14-36, KPF 14-64, KPMR 745, KPMR 851, KPMR 910, } \\
\text { KPMR 928, KPMR 935, NDP 11-101, NDP 11-102, NDP 14-11, NDPD 2017-06, NDPT 2017-04, NGSN-10, } \\
\text { NGSN-11, NGSN-13, NGSN-14, NGSN-3, NGSN-4, NGSN-5, NGSN-6, NGSN-8, NGSN-9, Pant P-125, Pant P- } \\
\text { 180, Pant P-184, Pant P-186, Pant P-195, Pant P-200, Pant P-2009-3, Pant P-207, Pant P-215, Pant P-217, Pant P- } \\
\text { 222, Pant P-223, Pant P-247, Pant P-250, Pant P-347, Pant P-365, Pant P-367, Pant P-375, Pant P-38, Pant P-397, } \\
\text { Pant P-399, Pant P-402, Pant P-42, PH-1, RFP 11-09, RFP 2009-2, RFP 2010-11, RFP 2010-3, RFP 2011-1, RFP } \\
\text { 72, RFPG 112, RFPG 114, SKUAP-8, SPC101, VL-201, VL-202, VL-55, VL-56, VL-58, V L-66, VL-67 }\end{array}$ & 126 \\
\hline & & Present:9 & None & 0 \\
\hline \multirow[t]{2}{*}{20.} & \multirow[t]{2}{*}{ Seed parchment } & Absent: 1 & $\begin{array}{l}\text { 20/1062, Aman, Ambika, DDR-36, DDR-4, DMR-7, EC 398601, EC 412882, HFP 0110, HFP 0130, HFP 0145, } \\
\text { HFP 0149, HFP 1010, HFP 1024, HFP 1036, HFP 1104, HFP 1107, HFP 1120, HFP 1125, HFP 1129, HFP 1130, } \\
\text { HFP 1132, HFP 1137, HFP 1140, HFP 1201, HFP 1302, HFP 1306, HFP 1307, HFP 1314, HFP 1315, HFP 1405, } \\
\text { HFP 1425, HFP 1428, HFP 1463, HFP 2008, HFP 530B, HFP 9106, HFP 920, HFP 9426, HFP 9907A, HFP } \\
\text { 9907B , HUDP 1301, HUDP 1302, HUDP 1711, HUDP 1715, HUPT 1701, IP2K 83-1, IPF 11-13, IPF 11-15, IPF } \\
\text { 17-18, IPF 17-19, IPF 5-19, IPFD 12-2, IPFD 12-8, IPFD 13-14, IPFD 13-2, IPFD 17-2, IPFD 17-6, IPFD 2014- } \\
\text { 11, KFP 12-4, KPF 1024, KPF 115, KPF 14-35, KPF 14-36, KPF 14-64, KPMR 745, KPMR 851, KPMR 910, } \\
\text { KPMR 928, KPMR 935, NDP 11-101, NDP 11-102, NDP 14-11, NDPD 2017-06, NDPT 2017-04, NGSN-10, } \\
\text { NGSN-11, NGSN-13, NGSN-14, NGSN-3, NGSN-4, NGSN-5, NGSN-6, NGSN-8, NGSN-9, Pant P-125, Pant P- } \\
\text { 180, Pant P-184, Pant P-186, Pant P-195, Pant P-200, Pant P-2009-3, Pant P-207, Pant P-215, Pant P-217, Pant P- } \\
\text { 222, Pant P-223, Pant P-247, Pant P-250, Pant P-347, Pant P-365, Pant P-367, Pant P-375, Pant P-38, Pant P-397, } \\
\text { Pant P-399, Pant P-402, Pant P-42, PH-1, RFP 11-09, RFP 2009-2, RFP 2010-11, RFP 2010-3, RFP 2011-1, RFP } \\
\text { 72, RFPG 112, RFPG 114, SKUAP-8, SPC101, VL-201, VL-202, VL-55, VL-56, VL-58, V L-66, VL-67 }\end{array}$ & 126 \\
\hline & & Present:9 & None & 0 \\
\hline
\end{tabular}


Out of three only two classes of pod intensity of green colour were observed in our set of accessions, out of which 3 genotypes bore light green pods (HFP 0130, HFP 1306 and Pant P-200 and remaining 123 were having green coloured pods.

Measurement of plant height grouped 5 genotypes as medium (HFP 1306, KPMR 928, Pant P-2009-3, Pant P-223, VL-201) and 121 as tall. Mehrani, (2002) and Singh et al., (2014) observed maximum variation for plant height in fieldpea genotypes and Bhuvaneswari et al., (2017) observed about $59 \%$ of the genotypes as dwarf with plant height $<60 \mathrm{~cm}$. Visual observation of seed shape revealed 91 genotypes were having spherical shaped seeds, 16 genotypes were having cylindrical and the remaining 19 genotypes were having dimpled seed shape, however, Singh et al., (2014) observed all the three classes of seed shape in their study.

In our genotypes, seed surface was observed smooth in 52, wrinkled in 74 genotypes which conform to the results of Singh et al., (2014). As far as seed cotyledon colour is concerned, only two categories i.e. yellow (95 genotypes) and green (31 genotypes) were observed. Bhuvaneswari et al., (2017) while characterizing fifty one field pea genotypes observed green color testa in majority of the genotypes and they reported both types of seed surface.

DUS guidelines for fieldpea (100-seed weight) classified genotypes into three groups viz., small $(<15 \mathrm{~g})$, medium $(15-20 \mathrm{~g})$ and large $(>20 \mathrm{~g})$. In our study, 41 genotypes were observed small, 82 genotypes were observed medium and three genotypes were observed large viz., HFP 1125, KPF 14-35, RFPG 114. Seed testa mottling was not observed in any of the genotype studied. Whereas, for seed parchment only one group was observed i.e. in all the genotypes seed parchment was absent.
From the study it can be concluded that the characters that show negligible environmental influence should only be relied upon for characterization because other quantitative traits are highly influenced by the environment and may show variation with change in environment. On the basis of present DUS characterization, these genotypes were evaluated and grouped into different categories for each character and which can serve as reference and the can be effectively used for identification and grouping of genotypes. Additionally, these genotypes can be used in varietal improvement programme depending upon the character specific objectives.I

\section{References}

Anonymous 2007. Guidelines for the Conduct of test for Distinctiveness, Uniformity and Stability on Fieldpea (Pisum sativum L.) PPV and FRA, Government of India, 2007, pp 1-9.

Anonymous 2019. Project Coordinators' Report, AICRP on MULLaRP (Rabi Crops), IIPR, Kanpur.

Bhuvaneswari, S., Sharma, S.K., Punitha, P., Shashidhar, K.S., Naveenkumar, K.L. and Prakash, N. 2017. Evaluation of morphological diversity of field pea [Pisum sativum subsp. arvense (L.)] germplasm under sub-tropical climate of Manipur. Legume Res. 40: 215-223.

Dixit, G.P., Katiyar, P.K. and Singh, B.B. 2010. Morphological characterization of Indian pea varieties. Journal of Food Legumes, 23(1): 25-29.

Duke, J.A. 1981. Hand book of Legumes of World Economic Importance. Plenum Press, New York, 199-265.

Mehrani, P. 2002. Genetic diversity in local and exotic pea (Pisum sativum L.) germplasm for morphological traits and SDS-PAGE markers. M. Phil. Dissertation, Quaid-e-Azam University, Islamabad. 
Mihailovic and Mikic 2010. Novel directions of breeding annual feed legumes in Serbia. Proceedings of XII International symposium on forage crops of Republic of Serbia, Krusevac, Serbia, 1: 81-90.

Nawab, N.N., Subhani, G.M., Mahmood, K., Shakil, Q. and Saeed, A. 2008. Genetic variability, correlation and path analysis studies in garden pea (Pisum sativum L.). Journal of Agricultural Research 46(4): 333-340.

Ouafi, L., Alane, F., Rahal-Bouziane, H. and Abdelguerfi, A. 2016. Agromorphological diversity within fieldpea (Pisum sativum L.) genotypes. African Journal of Agricultural Research; 11(40): 4039-404.

Singh, B., Chaubey, T., Upadhyay, D.K., Jha, A. and Pandey, S.D. 2014.
Morphological characterization of vegetable pea (Pisum sativum L. spp. Hortense) genotypes and their application for distinctiveness, uniformity and stability testing. Legume Research-An International Journal, 37(5): 547-551.

Tyagi, N., Singh, A.K., Rai, V.P., Kumar, S. and Srivastava, C.P. 2012. Genetic variability studies for lodging resistance and yield attributes in pea (Pisum sativum L.). Journal of Food Legumes, 25(3): 179-182.

Yadav, R.D.S., Purushottam, M.G., Bhati, J. and Yadav, P. 2019. Diagnostic characteristics of fieldpea varieties. International Journal of Chemical Studies, 7(4): 1701-1702.

\section{How to cite this article:}

Theint Theint Tun, Ravika, Rajesh Yadav and Suman. 2020. DUS Characterization of Field Pea Genotypes (Pisum sativum L.) Using Morphological Markers. Int.J.Curr.Microbiol.App.Sci. 9(11): 1978-1992. doi: https://doi.org/10.20546/ijcmas.2020.911.235 